\title{
VARIOUS LOCAL GLOBAL PRINCIPLES FOR ABELIAN GROUPS
}

\author{
George Peschke ${ }^{1}$ and Peter Symonds ${ }^{2}$
}

\begin{abstract}
We discuss local global principles for abelian groups by examining the adjoint functor pair obtained by (left adjoint) sending an abelian group $A$ to the local diagram $\mathcal{L}(A)=\left\{\mathbb{Z}_{(p)} \otimes A \rightarrow \mathbb{Q} \otimes A\right\}$ and (right adjoint) applying the inverse limit functor to such diagrams; $p$ runs through all integer primes. We show that the natural map $A \rightarrow \lim \mathcal{L}(A)$ is an isomorphism if $A$ has torsion at only finitely many primes. If $A$ is fixed we answer the genus problem of identifying all those groups $B$ for which the local diagrams $\mathcal{L}(A)$ and $\mathcal{L}(B)$ are isomorphic. A similar analysis is carried out for the arithmetic systems $\mathcal{S}(A)=\left\{\mathbb{Q} \otimes A \rightarrow \mathbb{Q} \otimes A^{\wedge} \leftarrow A^{\wedge}\right\}$ and the local systems $\left\{\mathbb{Q} \otimes A \rightarrow \mathbb{Q} \otimes\left(\Pi \mathbb{Z}_{(p)} \otimes A\right) \leftarrow \Pi\left(\mathbb{Z}_{(p)} \otimes A\right)\right\}$. The delicate relationship between the various adjoint functor pairs described above is explained.
\end{abstract}

\section{Introduction}

Given an abelian group $A$ it is often easier to work with the localization of $A$ at a prime $p$ than with $A$ itself. Thus one would like to analyze the localizations of $A$, one prime at a time, and then recover information about $A$ from its local data. For this reason we consider two methods of determining a group from local data.

I. We associate to an abelian group $A$ the inverse local diagram $\mathcal{L}(A):=\left\{r_{p}: A_{(p)} \rightarrow A_{\emptyset}\right\}$, where $A_{(p)}$ is the $p$-localization of $A$ and $r_{p}$ rationalizes. Specializing a result of $[7]$, we see that the natural map $c: A \rightarrow \lim \mathcal{L}(A)$ is an isomorphism if and only if $A$ has torsion at only finitely many primes.

\footnotetext{
${ }^{1}$ Supported by NSERC of Canada

${ }^{2}$ Partially supported by NSERC of Canada
} 
II. We associate to an abelian group $A$ the inverse local system $\mathcal{L S}(A):=\left\{A_{\emptyset} \rightarrow\left(\Pi A_{(p)}\right)_{\emptyset} \leftarrow\left(\Pi A_{(p)}\right)\right\}$. A result of [5] says that the natural map $c: A \rightarrow \lim \mathcal{L S}(A)$ is always an isomorphism.

Using results like I and II to obtain information about $A$ has a long tradition; see [4], [6], [10]. However, our foundation in I is much stronger than its counterpart in earlier expositions where the abelian group $A$ is assumed to be finitely generated. As a consequence we obtain new insight into some old questions related to the notion of the "genus" of a group.

We define the $\mathcal{L}$-genus of a group $A$ to consist of all isomorphism classes of groups $B$ with $\mathcal{L}(A) \cong \mathcal{L}(B)$. Thus the $\mathcal{L}$-genus measures the extent to which the local data in $\mathcal{L}(A)$ fail to determine $A$ uniquely. In section 3 , we show how the $\mathcal{L}$-genus can be calculated in terms of invariants familiar from homological algebra.

If we compare the recovery results I and II we see that the extent to which the local data determine a group depends crucially on the means used to splice the local data together. In section 6 we explain the relationship between the categories of local systems and local diagrams. In particular we explain which additional structural ingredient makes local systems a more powerful recovery tool than local diagrams.

Closely related to localization at a prime $p$ is $p$-completion. In fact, both processes have the same kernel but completion has a more structure enhancing quality. We carry out a similar development for completions as well. Then we show that the categories of local systems and complete systems are isomorphic.

Many of our methods and results can be extended in several directions. Firstly, to nilpotent groups and, secondly to algebras over a Dedekind domain. In section 7 we indicate, without proof, the nature of such extensions to Dedekind domains.

\section{Preliminaries}

We begin by collecting the necessary definitions and properties of $P$ localization of groups. For details, see [3], [4], [6]. Given a set of integral primes $P$, let $\mathbb{Z}_{P}$ denote the set of rational numbers whose denominator is not divisible by any $p \in P$. Thus $\mathbb{Z}_{\emptyset}$ denotes the rationals $\mathbb{Q}$. If $P$ consists of a single prime $p$ we write $\mathbb{Z}_{(p)}$ for $\mathbb{Z}_{P}$.

A homomorphism of nilpotent groups $e: G \rightarrow H$ is said to $P$-localize if and only if

(i) $H$ is a $P$-local group; i.e. for every prime $p \notin P$, the function $H \ni h \mapsto h^{p} \in H$ is a bijection;

(ii) the kernel of $e$ consists of all those torsion elements of $G$ whose order has no divisior in $P$; 
(iii) for every $h \in H$ there exists an integer $n$ with no divisor in $P$ such that $h^{n} \in \operatorname{im}(e)$.

Given $G$, the $P$-localizing homomorphism $e: G \rightarrow H$ is completely determined by $P$. So we write $e_{P}: G \rightarrow G_{P}$. The functor $G \mapsto G_{P}$ is exact and idempotent. Moreover, $e_{P}$ has a nonincreasing effect on the order of nilpotency of a given group. Every homomorphism from $G$ to a $P$-local group factors uniquely through $e_{P}$. This is called the universal property of $P$-localization. In particular, if $P \subset Q$ then $e_{P}$ factors uniquely through $e_{Q}$.

We turn to formal completions as introduced by Sullivan [9]. The $p$-adic integers $\mathbb{Z}_{p}^{\wedge}$ are given as the inverse limit of the system $\cdots \rightarrow$ $\mathbb{Z} / p^{2} \rightarrow \mathbb{Z} / p \rightarrow 0$. Formal $p$-completion of an abelian group $A$ is given by $A_{p}^{\wedge}:=A \otimes \mathbb{Z}_{p}^{\wedge}$. This is an exact functor because $\mathbb{Z}_{p}^{\wedge}$ is flat. The $p$-adic rationals can be obtained as $\mathbb{Q}_{p}^{\wedge}=\mathbb{Q} \otimes \mathbb{Z}_{p}^{\wedge}$. The completion of $\mathbb{Z}$ is $\mathbb{Z}^{\wedge}=\prod_{p} \mathbb{Z}_{p}^{\wedge}$ and the formal completion of an abelian group $A$ is $A^{\wedge}=A \otimes \mathbb{Z}^{\wedge}$. From [8] we know that $A_{p}^{\wedge}$ is the inverse limit of the system of quotients of $A$ which are $p$-torsion and that $A^{\wedge}$ is the inverse limit of the system of all torsion quotients of $A$.

\section{Properties of localizing and completing homomorphisms}

We make frequent use of the fact that the natural homomorphism from an abelian group to the product of its $p$-localizations or its $p$-completions is a monomorphism whose cokernel is a rational vector space. Results of this nature are collected in this section.

1.1. Lemma. The homomorphism e :A $\rightarrow \prod A_{(p)}$, whose $p$-th coordinate map is p-localization, is a monomorphism whose cokernel is a rational vector space.

Proof: The monomorphism part follows from (ii) in section 0 and is well known; see e.g. [6]. We investigate the cokernel of $i$ using the commutative diagram below. 


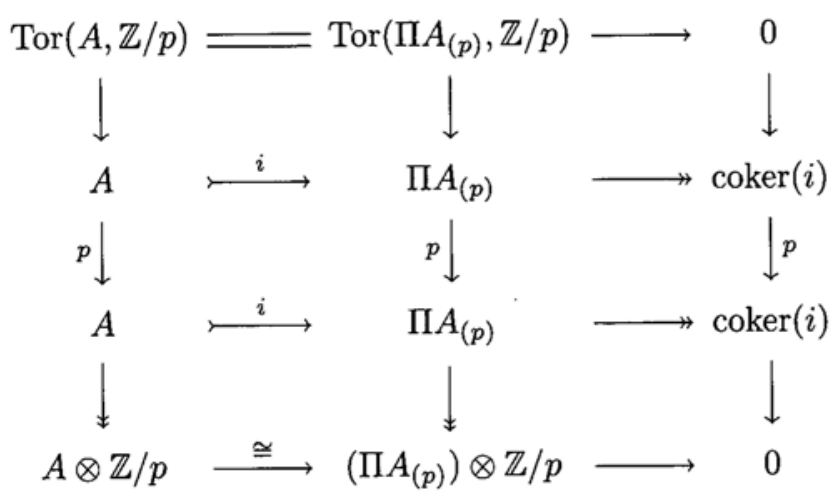

As the rows and the left two colums are exact, so is the right hand col$u m n$. Thus multiplication by any prime $p$ is an isomorphism on coker $(i)$. So $\operatorname{coker}(i)$ is a rational vector space.

1.2. Lemma. The natural maps $\mathbb{Z}_{(p)} \hookrightarrow \mathbb{Z}_{p}^{\wedge}$ and $\mathbb{Z} \hookrightarrow \mathbb{Z}^{\wedge} \cong \prod_{p} \mathbb{Z}_{p}^{\wedge}$ induce natural monomorphisms

$$
A_{(p)} \longmapsto A_{p}^{\wedge} \quad \text { and } \quad A \longmapsto A^{\wedge}
$$

whose cokernels are rational vector spaces.

Proof: We show first that $\mathbb{Z}_{p}^{\wedge} / \mathbb{Z}_{(p)}$ is a rational vector space. Consider the commuting diagram below.

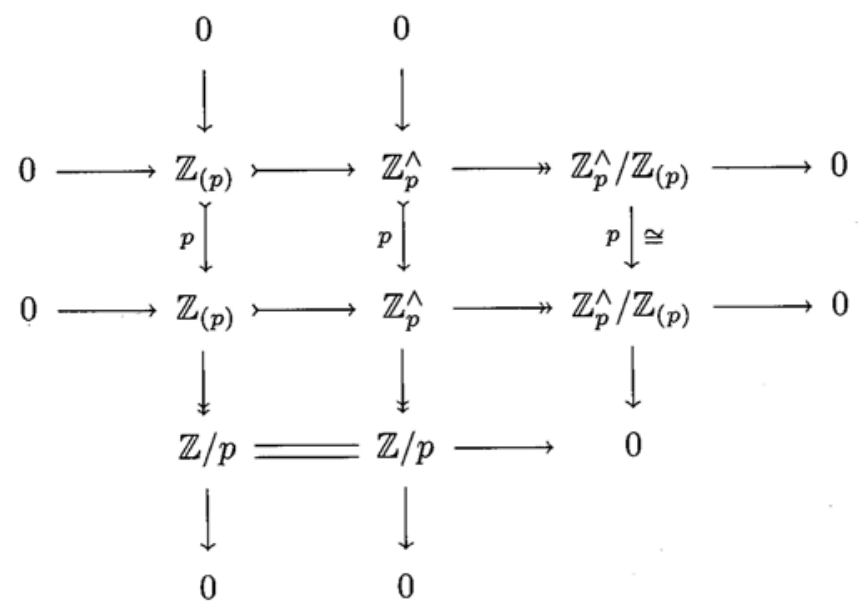




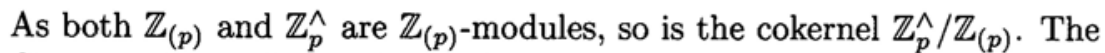
diagram above shows that multiplication by $p$ on this cokernel is an isomorphism. Therefore we have the exact sequence

$$
A_{(p)} \rightarrow A_{p}^{\wedge} \rightarrow A_{p}^{\wedge} / A_{(p)} \cong A \otimes\left(\mathbb{Z}_{p}^{\wedge} / \mathbb{Z}_{(p)}\right) .
$$

The claim follows because $\mathbb{Z}_{p}^{\wedge} / \mathbb{Z}_{(p)}$ is a $\mathbb{Q}$-module. For $A^{\wedge} / A$, the same method applied to

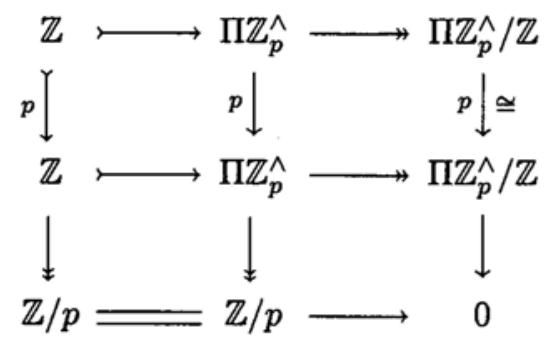

shows that $A^{\wedge} / A$ is a rational vector space.

1.3. Remark. The cokernel of the inclusion $\otimes_{p} \mathbb{Z}_{p}^{\wedge} \rightarrow \prod_{p} \mathbb{Z}_{p}^{\wedge}$ is a rational vector space.

Proof: The splitting $\prod_{p} \mathbb{Z}_{p}^{\wedge} \cong \mathbb{Z}_{p}^{\wedge} \oplus \prod_{q \neq p} \mathbb{Z}_{q}^{\wedge}$ shows that multiplication by $p$ on $\Pi \mathbb{Z}_{p}^{\wedge}$ has cokernel $\mathbb{Z} / p$. Now the proof can be completed as in the argument of the previous result.

\section{Facts about fiber squares}

Our main tool to identify pullback (fiber square) diagrams is the following lemma.

2.1. Lemma. Suppose $f: A \rightarrow B$ is a homomorphism between abelian groups whose kernel is a rational vector space and whose cokernel is torsion free. Then the diagram below is a pullback diagram in the category of all groups.

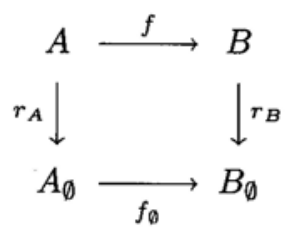


Proof: The lemma will follow after we have shown that, for $\alpha \in A_{\emptyset}$ and $b \in B$ with $f_{\emptyset}(\alpha)=r_{B}(b)$, there exists $a \in A$ with $f(a)=b$ and $r_{A}(a)=\alpha$.

Let $K=\operatorname{ker}(f)$. This is a rational vector space. So we get a splitting $A=K \oplus A_{1}$, and the restriction of $f$ to $A_{1}$ is an isomorphism to $\operatorname{im}(f)$. Now pick $n \geq 1$ and $a^{\prime} \in A$ such that $n \alpha=r_{A}\left(a^{\prime}\right)$. This yields

$$
r_{B} f\left(a^{\prime}\right)=f_{\emptyset} r_{A}\left(a^{\prime}\right)=n f_{\emptyset}(\alpha)=n r_{B}(b),
$$

and so $n b-f\left(a^{\prime}\right) \in \operatorname{ker}\left(r_{B}\right)=T[B]$, the torsion subgroup of $B$. As $\operatorname{coker}(f)$ is torsion free, there exists a torsion element $t \in A_{1}$ with $f(t)=$ $n b-f\left(a^{\prime}\right)$. Thus we have

$$
\begin{aligned}
r_{A}\left(t+a^{\prime}\right) & =r_{A}\left(a^{\prime}\right)=n \alpha \\
f\left(t+a^{\prime}\right) & =f(t)+f\left(a^{\prime}\right)=n b .
\end{aligned}
$$

Thus $n b \in \operatorname{im}(f)$ and, hence, $b \in \operatorname{im}(f)$; otherwise coker $(f)$ is not torsion free. So $b=f\left(a_{1}\right)$, for some $a_{1} \in A_{1}$. On the other hand,

$$
f_{\emptyset}(\alpha)=r_{B}(b)=r_{B}\left(f\left(a_{1}\right)\right)=f_{\emptyset}\left(r_{A}\left(a_{1}\right)\right)
$$

and, hence, $\left(\alpha-r_{A}\left(a_{1}\right)\right) \in \operatorname{ker}\left(f_{\emptyset}\right)=r_{A}(K)$. So let $r_{A}(k)=\alpha-r_{A}\left(a_{1}\right)$, for some $k \in K$ and set $a:=k+a_{1}$. By design, $f(a)=f\left(a_{1}\right)=b$ and $r_{A}(a)=\alpha$.

2.2. Corollary. For every abelian group $A$ the diagram below is a fiber square.

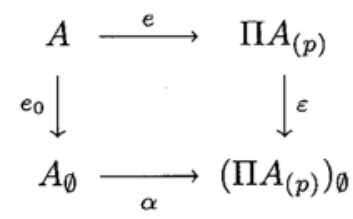

Here $e$ is the homomorphism whose $p$-th coordinate is the p-localization map of $A$. The rest of the diagram is obtained by rationalizing $e$.

Proof: This follows from 1.1 and 2.1 .

2.3. Remark. Corollary 2.2 has been shown by Hilton and Mislin [5] by different arguments. In fact they also show that (D) is a push-out diagram in the category of abelian groups. 
2.4. Proposition. For every abelian group $A$, there are fiber squares
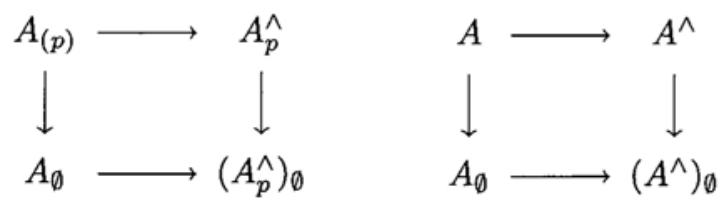

Proof: Use 1.2 and 2.1.

\section{The genus of an $\mathcal{L}$-diagram of abelian groups}

Let $\mathcal{L}$ denote the diagram of localizing functors

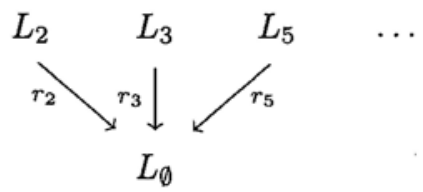

where $L_{p}$ denotes localization at the prime $p$ and $L_{\emptyset}$ denotes rationalization.

3.1. Definition. A diagram $\underline{A}=\left\{r_{p}: A_{p} \rightarrow A_{0}\right\}$ of abelian groups modeled on $\mathcal{L}$ is an $\mathcal{L}$-diagram if

(i) $A_{p}$ is a $p$-local group;

(ii) $A_{0}$ is a rational vector space;

(iii) for each prime $p, r_{p}: A_{p} \rightarrow A_{0}$ rationalizes.

The $\mathcal{L}$-diagrams form a category $\mathcal{L} \mathcal{A B}$. "Inverse limit" is a covariant functor $\mathcal{L} \mathcal{A B} \rightarrow \mathcal{A B}$ : it is right adjoint to the functor $\mathcal{A B} \rightarrow \mathcal{L A B}$ which sends an abelian group $A$ to the $\mathcal{L}$-diagram $\mathcal{L}(A):=\left\{L_{p} A \rightarrow L_{\emptyset} A\right\}$. The $\mathcal{L}$-genus of an $\mathcal{L}$-diagram $\underline{A}$ consists of all isomorphism classes of abelian groups $A$ with $\mathcal{L}(A) \cong \underline{A}$, and two groups $A$ and $B$ belong to the same $\mathcal{L}$-genus if $\mathcal{L}(A) \cong \mathcal{L}(B)$. In this section we relate the genus of an abelian group to invariants familiar from homological algebra.

3.2. Theorem. Let $\underline{A}=\left\{r_{p}: A_{p} \rightarrow A_{0}\right\}$ be an $\mathcal{L}$-diagram of abelian groups. Put $I:=\cap \operatorname{Im}\left(r_{p}\right)$ and suppose that $\underline{A}$ satisfies the condition

$$
I \hookrightarrow A_{0} \text { induces an isomorphism } I_{\emptyset} \rightarrow A_{0} \text {. }
$$


Let $T[\underline{A}]$ be the direct sum of the torsion subgroups $T\left[A_{p}\right]$ of $A_{p}$ and denote the product of the $T\left[A_{p}\right]$ by $\Pi[\underline{A}]=\varliminf_{\longleftarrow}\left\{T\left[A_{p}\right] \rightarrow A_{0}\right\}$. Set $\phi[\underline{A}]=$ $\lim _{\longleftarrow}\left\{A_{p} / T\left[A_{p}\right] \rightarrow A_{0}\right\}$. Then the following hold

(i) The sequence $\Pi[\underline{A}] \stackrel{i}{\leftrightarrow} \underline{A} \stackrel{q}{\rightarrow} \phi[A]$ is exact.

(ii) An abelian group $A$ belongs to the $\mathcal{L}$-genus of $\underline{A}$ if and only if there is a commutative diagram with exact rows.

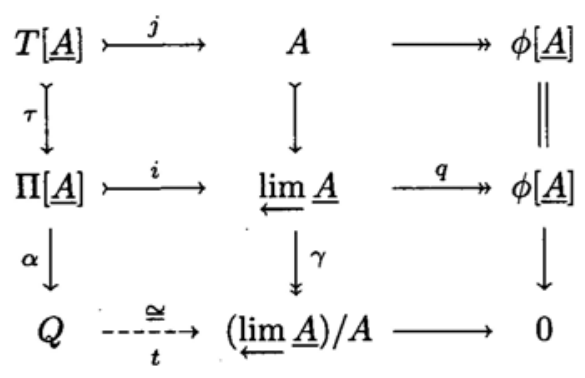

Proof: All claims pertaining to the top two rows of the diagram are special cases of 7.2 in [7]. We sketch the argument for completeness' sake.

The sequence $\Pi[\underline{A}] \stackrel{i}{\longmapsto} \lim _{\longleftarrow} \underline{A} \stackrel{q}{\rightarrow} \phi[\underline{A}]$ is the inverse limit of the diagram of short exact sequences $\left\{\left(T\left[A_{p}\right] \mapsto A_{p} \rightarrow A_{p} / T\left[A_{p}\right]\right) \rightarrow A_{0}\right\}$. Thus the inverse limit sequence is exact in the left hand and center positions, using general properties of inverse limits; see [1]. Moreover, $q$ is onto because $I=\phi[\underline{A}]$ and $\lim _{\longleftarrow} \underline{A} \rightarrow I$ is onto.

Next show that, given an abelian group $B$, the image of the rationalizing map $B \rightarrow B_{\emptyset}$ coincides with the intersection of the images $B_{(p)} \rightarrow B_{\emptyset}$. This implies the claim in the case where all the groups $A_{p}$ are torsion free because $\phi[\underline{A}]=I$.

In general, if $A$ satisifes $\mathcal{L}(A) \cong \underline{A}$, we see that $A / T[A] \cong \phi[\underline{A}]$ from the torsion free case. The maps from the top row to the middle row come from the universal property of inverse limits. Conversely, if $A$ is an extension as in the diagram we get commuting diagrams

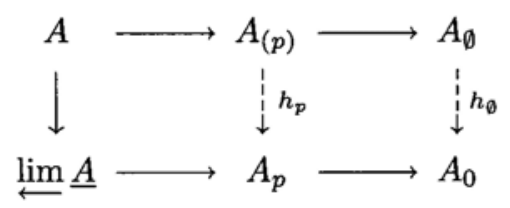


from the universal property of $p$-localization. Moreover, $h_{p}$ restricts to an isomorphism between torsion subgroups and induces an isomorphism between torsion free quotients. Thus $h_{p}$ is an isomorphism by the 5-lemma.

In either case, the map $t$ exists because $\operatorname{Ker}(\gamma)$ contains $i(\operatorname{Ker}(\alpha))$. The bottom row is seen to be exact by regarding the vertical arrows in the diagram as an exact sequence of chain complexes.

We use Theorem 3.2 to relate the genus of an $\mathcal{L}$-diagram $\underline{A}$ to invariants from homological algebra.

The homomorphism $\tau:(T:=T[\underline{A}]) \rightarrow(\Pi[\underline{A}]=: \Pi)$ induces the map

$$
\tau_{*}: \operatorname{Ext}(\phi, T) \rightarrow \operatorname{Ext}(\phi, \Pi)
$$

by taking the pushout as indicated.

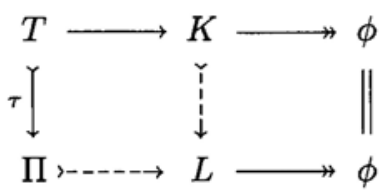

Let $\lambda \in \operatorname{Ext}(\phi, \Pi)$ denote the class corresponding to the extension $\Pi \hookrightarrow$ $\stackrel{\lim }{\longleftarrow} \underline{A} \rightarrow \phi$.

3.3. Theorem. If an $\mathcal{L}$-diagram of abelian groups satisfies condition $(*)$ of 3.2 then the elements of the $\mathcal{L}$-genus of $\underline{A}$ are in bijective correspondence with $\left(\tau_{*}\right)^{-1}(\lambda)$ modulo the action of the subgroup of $\operatorname{Aut}(\phi) \times \operatorname{Aut}(T)$ which stabilizes $\lambda$.

Proof: Every extension $B$ of $\phi$ by $T$ with $\mathcal{L}(B) \cong \underline{A}$ represents an element in $\tau_{*}^{-1}(\lambda)$ and every element in $\tau_{*}^{-1}(\lambda)$ determines a group $B$ with $\mathcal{L}(B) \cong \underline{A}$; by (3.2). Therefore, we determine which elements in $\tau_{*}^{-1}(\lambda)$ have isomorphic groups in the middle. Consider the exact sequence

(Hom-Ext)

$$
\begin{aligned}
0 & \rightarrow \operatorname{Hom}(\phi, T) \rightarrow \operatorname{Hom}(\phi, \Pi) \rightarrow \operatorname{Hom}(\phi, Q) \stackrel{\partial}{\rightarrow} \\
& \rightarrow \operatorname{Ext}(\phi, T) \underset{\tau_{*}}{\rightarrow} \operatorname{Ext}(\phi, \Pi) \rightarrow \operatorname{Ext}(\phi, Q)=0
\end{aligned}
$$

in which the last term is 0 because $Q$ is rational vector space (multiplication by an integer $n \neq 0$ in $\Pi$ is an isomorphism modulo $n$-torsion). Thus we see that $\tau_{*}$ is onto. Suppose $f: B_{1} \rightarrow B_{2}$ is an isomorphism between 
two extensions of $\phi$ by $T$. Then $f$ induces automorphisms $T[f]: T \rightarrow T$ and $\phi[f]: \phi \rightarrow \phi$ which make the diagram below commute

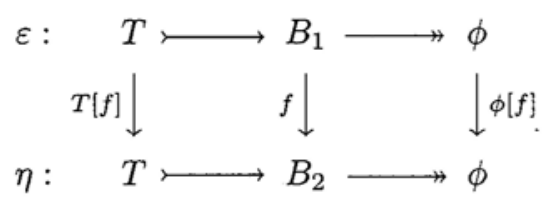

Hence $B_{1}$ is isomorphic to $B_{2}$ if and only if $\eta=\left(\phi[f]^{-1}\right)^{*} \circ T[f]_{*} \varepsilon$. This implies the claim.

We call an $\mathcal{L}$-diagram $\underline{A}$ realizable if it is of the form $\mathcal{L}(A)$ for some abelian group $A$. From the proof of 3.3 we see

3.4. Corollary. An $\mathcal{L}$-diagram $\underline{A}$ is realizable if and only if $\underline{A}$ satisfies condition 3.2(*).

The information pertaining to the $\mathcal{L}$-genus, which is encoded in the Hom-Ext sequence above, can be reinterpreted "geometrically" using an analogy with principle bundle classification. Let us consider all abelian groups whose torsion subgroup is a fixed group $T$. Associated with $T$ is the universal $T$-extension

$$
0 \rightarrow T \longmapsto \Pi \rightarrow Q \rightarrow 0
$$

where $\Pi$ is the product of the $p$-primary subgroups of $T$.

3.5. Theorem. Suppose the abelian groups $A$ and $A^{\prime}$ have torsion extensions

$$
0 \rightarrow T \mapsto A \rightarrow \phi \rightarrow 0
$$

and

$$
0 \rightarrow T \longmapsto A^{\prime} \rightarrow \phi \rightarrow 0
$$

Then $A$ and $A^{\prime}$ belong to the same $\mathcal{L}$-genus if and only if there exists a "classifying map" $c_{A^{\prime}}: \phi \rightarrow Q$ such that $\varepsilon_{A^{\prime}}=\varepsilon_{A}+\left(c_{A^{\prime}}\right)^{*}(\omega)$.

Proof: The boundary operator $\partial: \operatorname{Hom}(\phi, Q) \rightarrow \operatorname{Ext}(\phi, R)$ sends a map $c: \phi \rightarrow Q$ to $c^{*}(\omega)$. The claim follows from 3.3 using the exactness of the Hom-Ext sequence. 
3.6. Corollary. Suppose the element $\lambda \in \operatorname{Ext}(\phi, \Pi)$ in the previous proposition is 0 . Then the genus of $\underline{A}$ is in bijective correspondence with the cokernel of $\operatorname{Hom}(\phi, \Pi) \rightarrow \operatorname{Hom}(\phi, Q)$ modulo the action of $\operatorname{Aut}(\phi) \times$ $\operatorname{Aut}(T)$.

3.7. Remark. It is well known that $\operatorname{Ext}(\phi, T)=0$ for all torsion free $\phi$ if and only if $T$ is the direct sum of a divisible group $D$ and a group $T^{\prime}$ of finite exponent (i.e. $k T^{\prime}=0$, for a suitable $k \in \mathbb{N}$ ); see [2]. Consequently, a group $A$ whose torsion group $T$ is the direct sum of a divisible group and a group of finite exponent is itself isomorphic to $T \oplus A / T$. In particular, $A$ has trivial $\mathcal{L}$-genus.

\section{Examples}

4.1. Remark. The genus of the $\mathcal{L}$-diagram $\underline{A}=\{\mathbb{Z} / p \oplus \mathbb{Q} \stackrel{\text { project }}{\longrightarrow}$ $\mathbb{Q}\}$ is in bijective correspondence with the power set of the set of all primes.subject to the equivalence relation that $X \sim Y$ if and only if the symmetric difference of $X$ and $Y$ is finite.

Proof: Let $T$ be the sum of the groups $\mathbb{Z} / p$ and let $\Pi$ be the product of the groups $\mathbb{Z} / p$. Then the Hom-Ext sequence in the proof of 3.3 reads

$$
0=\operatorname{Hom}(\mathbb{Q}, \Pi) \rightarrow \operatorname{Hom}(\mathbb{Q}, Q) \rightarrow \operatorname{Ext}(\mathbb{Q}, T) \rightarrow \operatorname{Ext}(\mathbb{Q}, \Pi) .
$$

One group in the genus of $\underline{A}$ is $T \oplus \mathbb{Q}$. So $\lambda=0$. Moreover, $\operatorname{Hom}(\mathbb{Q}, \Pi)=$ 0 since no nonzero element of $\Pi$ is divisible. Consequently, the elements in the genus of $\underline{A}$ correspond bijectively with the orbits of $\operatorname{Hom}(\mathbb{Q}, Q) \cong$ $Q$ under the action of $\operatorname{Aut}(\mathbb{Q}) \times \operatorname{Aut}(T)$, where $\operatorname{Aut}(\mathbb{Q})$ acts as scalar multiplication on $Q$ and $\operatorname{Aut}(T)=\prod \operatorname{Aut}(\mathbb{Z} / p)$ acts on $T$ and on $\Pi$, hence on their quotient $Q$. We calculate these orbits: It can be seen that each $\operatorname{Aut}(\mathbb{Q})$-orbit is contained in an $\operatorname{Aut}(T)$-orbit. Thus it suffices to consider the action of $\operatorname{Aut}(T)$ on $Q$. Represent $\omega \in Q$ by $x \in \Pi$. Using the action of $\operatorname{Aut}(T)$, we can alter $x$ so that all its non-zero coordinates are 1. Let $X$ denote the set of primes corresponding to non-zero coordinates of $x$. Another element $y \in \Pi$ represents an element in the $\operatorname{Aut}(T)$-orbit of $\omega$ if and only if the symmetric difference of $X$ and $Y$ is finite. This implies the claim.

The argument just given is constructive in that it allows us to describe the group $A_{X}$ in the $\mathcal{L}$-genus of $\underline{A}$ corresponding to a given set $X$ of primes; compare 3.5: Let $x \in \Pi$ be the element with 1 's as the coordinates in $X$ and 0's everywhere else. Then $x$ represents an element in $Q$ and, hence, a homomorphism $c_{x}: \mathbb{Q} \rightarrow Q$. So $A_{X}$ is obtained via the pullback of the sequence

$$
T \longmapsto \Pi \rightarrow Q
$$


along $c_{x}$. Consequently, $A_{X}$ is isomorphic to the subgroup $\alpha^{-1}\left(\operatorname{im}\left(c_{x}\right)\right)$ of $\Pi$.

4.2. Example. Let $Q$ be a set of primes and put

$$
T(Q):=\bigoplus_{p \in Q} \mathbb{Z} / p \quad \text { and } \Pi(Q):=\prod_{p \in Q} \mathbb{Z} / p
$$

Now define $E(Q):=\Pi(Q) / T(Q)$. Then $B(Q):=T(Q) \oplus E(Q)$ has the same $\mathcal{L}$-genus as $\Pi(Q)$. To see this, note that $E(Q)$ is a rational vector space. Further, if $p \in Q$, we get an isomorphism $\Pi(Q)_{(p)} \cong \mathbb{Z} / p \oplus E(Q)$ from the splitting $\Pi(Q) \cong \mathbb{Z} / p \oplus \Pi_{q \in(Q-\{p\})} \mathbb{Z} / q$.

4.3. Remark. Two groups $A$ and $B$ are customarily defined to be in the same genus if $A_{(p)} \cong B_{(p)}$ for all primes $p$. This notion corresponds to the $\mathcal{K}$-genus associated with the diagram of localizing functor $L_{2} L_{3}$ $L_{5} \ldots$ (no arrows). The $\mathcal{K}$-genus differs from the $\mathcal{L}$-genus in that no coherence conditions are imposed amongst the localizing functors involved. As a consequence, it is rarely possible to recover an infinite group from its $\mathcal{K}$-diagram. This is illustrated in the following

4.4. Example. The number of isomorphism classes of abelian groups in the same $\mathcal{K}$-genus as $\mathbb{Z}$ is uncountable. To see this, choose integers $r_{p} \geq 0$ for each prime $p$, and let $A_{r}$ be the subgroup of $\mathbb{Q}$ generated by $\left\{p^{-r_{p}}\right\}$. Then $\left(A_{r}\right)_{(p)} \cong \mathbb{Z}_{(p)}$ for all $p$, but $A_{r} \cong A_{s}$ if and only if $r$ and $s$ differ at only finitely many primes; see [2]. The groups $A_{r}$ represent the entire incoherent $\mathcal{K}$-genus of $\mathbb{Z}$.

In contrast, the genus of the $\mathcal{L}$-diagram $\left\{p^{-r_{p}} \mathbb{Z}_{(p)} \hookrightarrow \mathbb{Q}\right\}$ consists of a single element, namely the group $A_{r}$; see theorems 3.2 and 3.3.

\section{5. $\mathcal{L}$-diagrams, $\mathcal{L}$-systems}

In this section we explain the relationship between various local global principles involving completion or localization.

5.1. Definition. A local (arithmetic) system $\mathcal{L S}$ consists of a $\mathbb{Z}_{(p)^{-}}$ module $B_{p}$, for each prime $p$, and a $\mathbb{Q}$-module $B_{0}$ together with rational isomorphisms $k_{p}: B_{p} \rightarrow B_{0}$ such that

$$
\operatorname{im}\left(B_{0} \rightarrow \Pi_{p}\left(B_{0}\right) \stackrel{\cong}{\rightarrow} \Pi\left(B_{p}\right)_{\emptyset}\right)<\operatorname{im}\left(\left(\Pi B_{p}\right)_{\emptyset} \rightarrow \Pi\left(B_{p}\right)_{\emptyset}\right) .
$$

5.2. Remark. The last condition ensures that the various isomorphisms $k_{p}$ can be assembled over a single map $c: B_{0} \rightarrow\left(\prod B_{p}\right)_{\emptyset}$ (not necessarily unique) according to the commuting diagram below. 


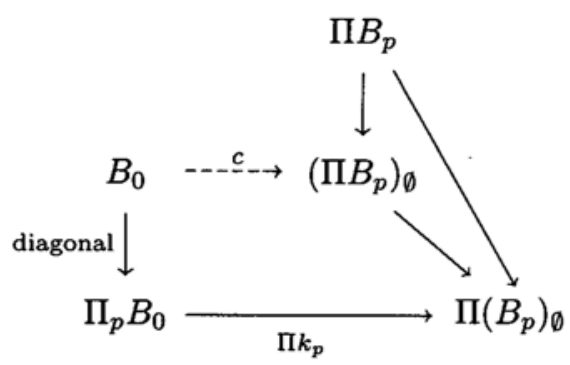

Thus we may form the category $\mathcal{L S}_{*}$ of local systems with assembly map c. An object of $\mathcal{L} \mathcal{S}_{*}$ is denoted by $\widetilde{B}_{*}$.

5.3. Lemma. Let $B$ denote the pullback of the local system

$$
\widetilde{B}_{*}=\left\{B_{0} \stackrel{c}{\rightarrow}\left(\Pi B_{p}\right)_{\emptyset} \leftarrow \Pi B_{p}\right\} .
$$

Then $\mathcal{L} \mathcal{S}_{*}(B) \cong \widetilde{B}_{*}$.

Proof: This follows from 2.2.

5.4. Remark. Two assembly maps $B_{0} \rightarrow\left(\prod B_{p}\right)_{\emptyset}$ differ by an element of $\operatorname{Hom}(B, Q)$, where

$$
Q:=\operatorname{ker}\left(\left(\Pi B_{p}\right)_{\emptyset} \rightarrow \Pi\left(B_{p}\right)_{\emptyset}\right) \cong \Pi T\left[B_{p}\right] / \oplus T\left[B_{p}\right] .
$$

Thus $c$ is unique if $\widetilde{B}_{*}$ has torsion at only finitely many primes.

5.5. Definition. A complete system $\widehat{C}$ consists of a $\mathbb{Z}_{p}^{\wedge}$-module $C_{p}$, for each prime $p$, and a $\mathbb{Q}$-module $C_{0}$ together with isomorphisms $v_{p}:\left(C_{0}\right)_{p}^{\wedge} \rightarrow\left(C_{p}\right)_{\emptyset}$ such that

(CS) $\quad \operatorname{im}\left(C_{0} \rightarrow \Pi_{p}\left(C_{0}\right)_{p}^{\wedge} \underset{\Pi v_{p}}{\stackrel{\cong}{\longrightarrow}} \Pi\left(C_{p}\right)_{\emptyset}\right)<\operatorname{im}\left(\left(\Pi C_{p}\right)_{\emptyset} \rightarrow \Pi\left(C_{p}\right)_{\emptyset}\right)$.

A morphism between complete systems is a commuting diagram

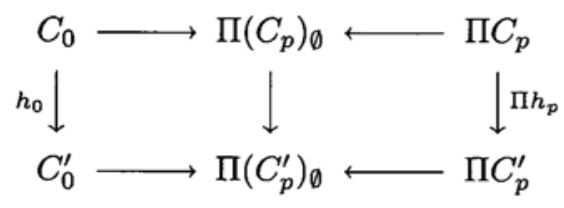

such that each $h_{p}$ is a $\mathbb{Z}_{p}^{\wedge}$-module homomorphism. 
5.6. Proposition. For any complete system $\widehat{C}_{*}$ with assembly map $c$ let $A$ be the pull-back

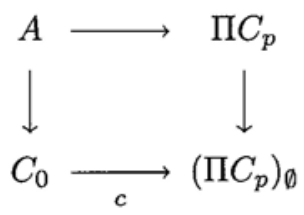

Then $\mathcal{C S}_{*}(A) \cong \widehat{C}_{*}$.

For the proof we need the following

5.7. Lemma. For any $\mathbb{Z}_{p}^{\wedge}$-module $C_{p}$ the natural homomorphism

$$
m:\left(C_{p}\right)_{p}^{\wedge} \ni b \oplus r \mapsto r b \in C_{p}
$$

has as its kernel a vector space over $\mathbb{Q}_{p}^{\wedge}$.

Proof: The kernel is a $\mathbb{Z}_{p}^{\wedge}$-module. To see that it is also a $\mathbb{Q}$-module notice that $m$ is split by the inclusion $C_{p} \rightarrow\left(C_{p}\right)_{p}^{\wedge}$ of 1.2 , whose cokernel is a rational vector space.

Proof of 5.6: There is a commutative diagram

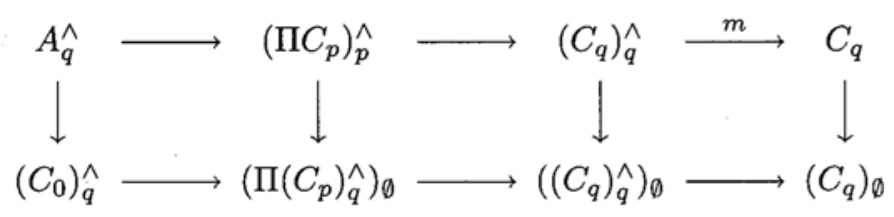

The three squares are fiber squares: the first because it is the completion of a fiber square and the other two by 2.1. Therefore the outer rectangle is a fiber square. Its bottom row is an isomorphism. So its top row is an isomorphism as well. We see that $A_{0} \cong C_{0}$ by rationalizing the pullback diagram defining $A$. Together these isomorphism give an isomorphism of diagrams.

With the same methods we prove

5.8. Lemma. If $A$ is defined as the pullback of $\sigma: B_{0} \rightarrow\left(B_{p}\right)_{\emptyset} \leftarrow B_{p}$, a part of a complete system, then $A_{\emptyset} \rightarrow\left(A_{p}^{\wedge}\right)_{\emptyset} \leftarrow A_{p}^{\wedge}$ is isomorphic to $\sigma$. 


\section{Summary of equivalences of categories}

In this section we provide a list of categories which are isomorphic with respect to functors used in a local global principle.

6.1. Theorem. For abelian groups we have the following equivalences of categories.

(i) $\{$ realizable local diagrams $\} \simeq\{$ local systems $\} \simeq\{$ complete systems $\}$ $\mathcal{L}_{r}$ $\mathcal{L S}$ $\mathcal{C S}$

(ii) $\{$ abelian groups $\} \simeq\{$ assembled local systems $\}$ $\mathcal{A B}$

$\mathcal{L S}_{*}$ $\simeq\{$ assembled complete systems $\}$ $\mathcal{C S}_{*}$

(iii) Let the subscript " $f$ " with any of the categories above denote "subcategory whose objects have torsion at only finitely many primes". Then

$$
\mathcal{A} \mathcal{B}_{f} \simeq\left(\mathcal{L}_{r}\right)_{f} \simeq \mathcal{L} \mathcal{S}_{f} \simeq\left(\mathcal{L} \mathcal{S}_{*}\right)_{f} \simeq \mathcal{C} \mathcal{S}_{f} \simeq\left(\mathcal{C} \mathcal{S}_{*}\right)_{f}
$$

The remainder of this section will be devoted to proving 6.1. All the functors giving these equivalences preserve the $f$-subcategories. So 6.1.iii will follow from the fact that $\mathcal{A} \mathcal{B}_{f} \simeq \mathcal{L}_{f}$, which is a corollary of 3.2 .

Proof of 6.1: We know that $\mathcal{A B} \simeq \mathcal{L S}_{*}$, by 5.3. Also $\mathcal{A B} \simeq \mathcal{C} \mathcal{S}_{*}$ by 5.6. Thus 6.1.ii is proved. A local diagram $\mathcal{L}=\left\{h_{p}: A_{p} \rightarrow A_{0}\right\}$ determines a local system $\mathcal{L S}$ with $B_{p}=A_{p}, B_{0}=A_{0}$ and $k_{p}=h_{p}$ and vice versa. We need to check that the realizability condition $I \hookrightarrow A_{0}$ induces an isomorphism $I \rightarrow A_{0}$, where $I:=\cap \operatorname{im}\left(r_{p}: A_{p} \rightarrow A_{0}\right)$, is equivalent to 5.1(LS), where $I:=\cap \operatorname{im}\left(h_{p}: A_{p} \rightarrow A_{0}\right)$.

Identify $A_{0}$ with its image in $\Pi\left(A_{p}\right)_{\emptyset}$ under the diagonal map. To see that realizability implies 5.1(LS), notice that $I:=\cap \operatorname{im} h_{p} \subset \Pi\left(A_{p}\right)_{\emptyset}$. Consequently, $I_{\emptyset}=A_{0}=A_{0} \cap \operatorname{im}\left(\Pi A_{p}\right)_{\emptyset}$.

Conversely, if the local system associated with the $\mathcal{L}$-diagram $\underline{A}$ satisfies 5.1(LS), choose a lift

$$
c: A_{0} \rightarrow\left(\Pi A_{p}\right)_{\emptyset} \quad \text { of } \quad A_{0} \rightarrow \Pi\left(A_{p} \oplus \mathbb{Q}\right),
$$

and define $A$ by the pullback of $A_{0} \underset{c}{\rightarrow} \prod\left(A_{p}\right)_{\emptyset} \leftarrow \prod A_{p}$. Now realizability of $\underline{A}$ follows from 5.3 .

To see that $\mathcal{L S} \simeq \mathcal{C S}$, suppose a local system $\widetilde{B}$ is given. Setting $C_{0}:=B_{0}$ and $C_{p}:=B_{p} \oplus \mathbb{Z}_{p}^{\wedge}$, we get a complete system. The condition 
5.5(CS) is satisfied because the map $B_{0} \rightarrow \prod\left(B_{p} \oplus \mathbb{Q}_{p}^{\wedge}\right)$ lifts to $B_{0} \rightarrow$ $\left(\prod\left(B_{p} \oplus \mathbb{Z}_{p}^{\wedge}\right)\right)_{\emptyset}$ according to the commuting diagram below.

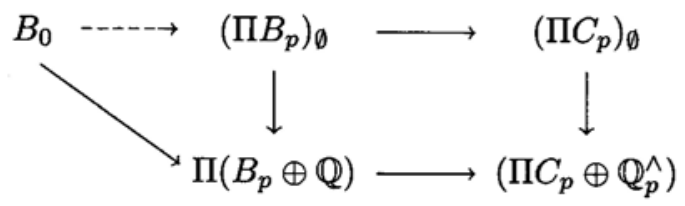

Conversely, given a complete system $\widehat{C}$, we construct a local system $\widetilde{B}$ by setting $B_{0}:=C_{0}$ and defining $B_{p}$ to be the pullback of $C_{0} \rightarrow\left(C_{p}\right)_{\emptyset} \leftarrow$ $B_{p}$; compare 2.4. To see that condition 5.1(LS) is satisfied, consider the commuting diagram below.

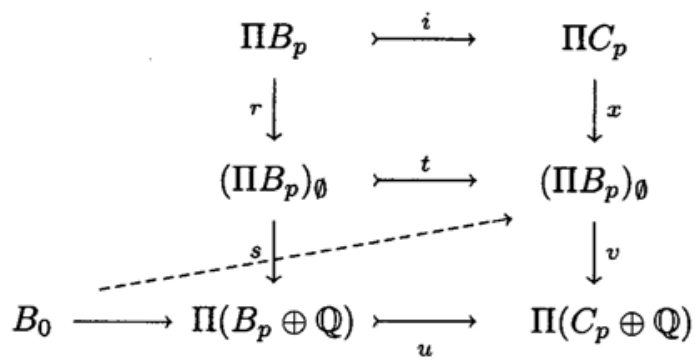

The outer rectangle is a fiber square since it is a product of fiber squares. Its rationalization is the bottom rectangle, which is, therefore, also a fiber square. Condition 5.1(LS) now follows using the universal property of pullbacks.

\section{Further remarks}

7.1. Remark. If we regard an abelian group as a $\mathbb{Z}$-module then there is an obvious generalization of this work to the case of modules over a Dedekind domain $R$, where localization, completion and torsion are all with respect to the prime ideals of $R$. The appropriate generalizations of all our results and their proofs remain valid.

Since our constructions are natural, most of them also apply to modules for an algebra $A$ over $R$ (localization etc. are still with respect to primes of $R$ ). Theorem 6.1 remains true but (3.4) may fail. The problem is that $\tau_{*}^{-1}(\lambda)$ in 3.3 may be empty when there is torsion at infinitely many primes. However, corollary 3.4 remains valid if every $A_{\emptyset}$-module is injective. This happens, for example, if $A$ is the group algebra of a finite group. 
7.2. Example. Let $C=\langle c\rangle$ be an infinite cyclic group. For each prime $p$, let $A_{p}=\mathbb{Z} / p \oplus \mathbb{Z}_{(p)}$ be a $\mathbb{Z}_{(p)}[C]$-module with action $c(x, y)=$ $(x+\bar{y}, y), x \in \mathbb{Z} / p, y \in \mathbb{Z}_{(p)}$ and $\bar{y}$ the image of $y$ in $\mathbb{Z} / p$. Let $A_{\emptyset}=\mathbb{Q}$ and let $r_{p}: A_{(p)} \rightarrow \mathbb{Q}$ be the usual inclusion of $\mathbb{Z}_{(p)}$ in $\mathbb{Q}$. This local diagram has a unique realization as an abelian group, namely $\left(\oplus_{p} \mathbb{Z} / p\right) \oplus$ $\mathbb{Z}$. However, the action of $C$ does not extend because $c(0,1)$ would have to have non-zero coordinate in every summand.

Theorem 6.1 can be generalized to nilpotent groups. We state the result. Its proof is completely parallel to our treatment of the abelian case. An additional technical quibble arises, however, from the fact that not every subgroup of a nilpotent group is normal. This is relevant, for example, in the nilpotent analogue of lemma 1.1.

7.3. Theorem. If $\mathfrak{C}$ is any of the categories above, let $\mathfrak{R C}$ denote the analogous category but with nilpotent objects. Then we have the following equivalences of categories.

(i) $\mathfrak{R F} \simeq \mathfrak{R L G}$;

(ii) $\mathfrak{R} \simeq$ the subcategory of $\mathfrak{R U L G}$ whose objects have a uniform bound on their order of nilpotency;

(iii) $\mathfrak{R}_{f} \simeq \mathfrak{R F}_{f} \simeq \mathfrak{R L G} \mathfrak{G}_{f} \simeq \mathfrak{R U L G} \mathfrak{G}_{f}$.

\section{References}

1. Bourbaki N., "Theory of Sets," Addison Wesley, Reading/Don Mills, 1968.

2. Fuchs L., "Infinite Abelian Groups II," Academic Press, New York, London, 1973.

3. Hilton P. J., Localization and cohomology of nilpotent groups, Math. Z. 132 (1973), 265-286.

4. Hilton P. J., "Nilpotente Gruppen und nilpotente Räume," Springer LNM 1053, Berlin/New York, 1984.

5. Hilton P. J. ANd Mislin G., Bicartesian Squares of Nilpotent Groups, Comment. Math. Helv. 50 (1975), 477-491.

6. Hilton P. J., Mislin G. AND Roitberg J., "Localization of Nilpotent Groups and Spaces," North-Holland, Amsterdam, 1975.

7. Peschke G., Localization and genus in group theory, Trans. AMS, to appear.

8. Peschke G. And Symonds P., Pro-torsion completions of abelian groups, J. Pure and Applied Algebra 88 (1993), 187-197.

9. Sullivan D., Geometric Topology I and II, MIT Notes, 1970. 
10. Sullivan D., Genetics of homotopy theory and the Adams conjecture, Ann. of Math. 100 (1974), 1-79.

George Peschke:

Department of Mathematical Sciences

University of Alberta

Edmonton

CANADA T6G 2G1
Peter Symonds:

Department of Mathematics

Northwestern University

Evanston, IIl.

60208 U.S.A.

Primera versió rebuda el 2 de Febrer de 1994, darrera versió rebuda el 16 de Maig de 1994 